\title{
Los Fondos de Identidad y el tercer espacio. Una estrategia de legitimación cultural y diálogo para la escuela intercultural
}

\author{
Funds of Identity and third space. A strategy of cultural legitimization \\ and dialogue for the intercultural school
}

\section{José Luis Lalueza ${ }^{a}$, Cristina Zhang-Yu ${ }^{b}$, Sarai García-Díaz, Sílvia Camps-Orfila ${ }^{b}$ David García-Romero ${ }^{b}$}

\author{
${ }^{a}$ Universitat Autònoma de Barcelona \\ Correo electrónico: Joseluis.lalueza@uab.cat \\ ${ }^{\mathrm{b}}$ Universitat Autònoma de Barcelona
}

\begin{abstract}
RESUMEN
La inclusión en la escuela de alumnos procedentes de la inmigración y de grupos racializados supone afrontar los retos planteados por las discontinuidades entre la cultura escolar y la familiar, indicadoras de unas relaciones de poder asimétricas por las que la cultura familiar y comunitaria del alumno no es legitimada en tanto que se mantiene fuera del aula e incluso es estigmatizada. En este trabajo se analiza una intervención con alumnos de secundaria basada en los Fondos de Identidad como estrategia para incorporar y legitimar los referentes personales de los alumnos con el objetivo de crear un tercer espacio en el que el diálogo intercultural es motor de los procesos de aprendizaje e inclusión escolar.
\end{abstract}

Palabras clave: Fondos de conocimiento, inmigración, educación intercultural, comunidades de práctica, educación artística

\begin{abstract}
The inclusion in the school of students from immigrant and racialized groups entails facing the challenges related with discontinuities between school and family culture, which are indicators of asymmetric power relations for which the student's family and community culture are not legitimized. It remains outside the classroom and is stigmatized. This paper analyzes an intervention with high school students based on Funds of Identity as a strategy to incorporate and legitimize the personal referents of the students. Our aim is to create a third space in which intercultural dialogue is the driving force of learning processes and school inclusion.
\end{abstract}

Key words: Funds of knowledge, immigration, intercultural education, communities of practices, artistic education 


\section{INTRODUCCIÓN}

Ya abandonando la segunda década del siglo XXI, vemos con claridad cómo las dinámicas del mundo globalizado, deslocalizando la producción y profundizando conflictos internacionales, llevan a agudizar los procesos migratorios que, conjuntamente con la diversidad ya presente en cada territorio (con sus hegemonías, mayorías y minorías) llevan a difuminar los límites culturales y a constituir una población visiblemente heterogénea.

Estas migraciones no solo aportan diversidad social, sino que constituyen también transformaciones en los sistemas de desigualdad y de exclusión (De Sousa Santos, 2003) que dan nuevas formas a discriminaciones y opresiones construidas históricamente. Situándonos concretamente en el plano de las diferencias culturales, podemos ver cómo se produce una transformación de los discursos basados en el esencialismo biológico en un nuevo esencialismo cultural, pero que igualmente marca una clara diferenciación entre la población autóctona y la migrante y/o racializada (identificada como ajena pese a ser nacida y socializada en el territorio que se encuentre), y que a través de esta diferenciación se esencializa en la diferencia, se estereotipa y se estigmatiza (Buraschi y Aguilar-Idáñez, 2017). En el marco de estas discriminaciones se producen discontinuidades culturales y conflictividad que a menudo se identifica como fruto de la diferencia en sí misma, cuando tiene más que ver con las relaciones de poder y las resistencias a los procesos que conducen hacia la asimilación o bien hacia la marginación de un grupo minoritario (Berry, 2001).

Es en este contexto de relaciones de poder en el que cobran significado las discontinuidades entre la práctica escolar y las prácticas familiares (Poveda, 2001). La escuela es una institución que responde a metas y valores hegemónicos cuyas prácticas encuentran el sentido en narrativas que comparten con las familias de clase media de la cultura hegemónica, pudiendo entrar en fuertes contradicciones con las narrativas que sustentan otros grupos (Sebastián, Gallardo y Calderón, 2016). Es en este último caso en el cual las discontinuidades en valores y metas dificultan la apropiación de la práctica escolar, así como la incorporación de los proyectos de vida escolares en las identidades de los alumnos, quienes transitan por esa institución ajenos a la misma e incluso en oposición a ella y sus valores.

La inclusión educativa es un proyecto ingenuo si no se tiene en cuenta la estigmatización y segregación histórica de grupos culturales y las relaciones de poder que estas implican. Es por ello que una escuela inclusiva, no segregadora, ha de ser necesariamente una escuela intercultural, en la que un posicionamiento explícito contra el racismo se construye de la mano de la legitimación de las voces y las prácticas de los grupos minorizados. La interculturalidad ha de suponer un diálogo real entre la hegemonía y las diferentes comunidades culturales (Buraschi y Aguilar-Idáñez, 2017) para lo que es necesario a) la legitimación de las diferentes personas como interlocutores válidos y b) la legitimación de diferentes epistemologías con sus diferentes formas de expresión.

Una vía que orienta en esa dirección es la estrategia que puso en marcha Luis Moll (González, Moll y Amanti, 1991), enfrentando la deslegitimación de la cultura propia de las familias hispanas en Arizona ante una escuela en la que se prohibía el uso del español. Nos referimos a los Fondos de Conocimiento ( $\mathrm{FdC}$ ), un proyecto que ha tenido un largo desarrollo internacional en las tres últimas décadas. Se trata de una estrategia de contextualización educativa que busca el reconocimiento de la validez de los conocimientos y prácticas culturales de las familias pertenecientes a grupos no hegemónicos. De esta 
manera, se estructura como una forma de investigación de los recursos epistémicos y sociales de cada núcleo familiar para posteriormente incorporarlos en la agenda educativa. La fuerza de esta estrategia recae primordialmente en la construcción de lazos de confianza y de mutuo conocimiento entre familia y escuela (Llopart y Esteban-Guitart, 2016) mediante la transformación de las relaciones de poder entre ambas.

Como derivación metodológica del mismo marco conceptual, los Fondos de Identidad (FdI) se orientan a situar al alumnado en el centro del diseño de prácticas educativas para generar aprendizajes significativos a la vez que se le interpela reconociendo la legitimidad de su voz y de los diversos referentes culturales en que pueda sustentarse.

Entendemos por FdI el conjunto de "recursos históricamente acumulados, culturalmente desarrollados y socialmente distribuidos" (Esteban-Guitart y Moll, 2014, p. 37) que toman significado y forman parte de cómo una persona se comprende y se construye. Estos recursos se manifiestan a través de espacios geográficos (lugares, países), prácticas identitarias (actividades significativas), fondos culturales (religión, grupo cultural u otras categorías sociales), fondos sociales (amistades, parientes, animales) y fondos institucionales (familia, iglesia, escuela). Mediante actividades y estrategias narrativas lingüísticas, plásticas y/o digitales (Díaz Barriga, López y Vázquez, 2018), el estudiantado indaga en su propio mundo, promoviendo una autorreflexión sobre quiénes son y qué elementos son los más significativos para sí mismos. Recientemente se han llevado a cabo estrategias con perspectiva de justicia social pedagógica mediante los FdI (Cammarota, 2014; Subero, Vila y Esteban-Guitart, 2015; Zipin, 2009) evidenciando la posibilidad que ofrece para construir propuestas críticas y transformadoras que problematicen las narrativas escolares hegemónicas (Sebastián, Gallardo y Calderón, 2016) y generen espacios para la producción de narrativas que pongan en valor las experiencias y saberes de las aprendices.

Desde nuestro punto de vista, la virtud de los FdI reside en su potencial para crear un tercer espacio en el aula. Kris Gutierrez (2008) desarrolla la idea de un tercer espacio como contexto de actividad que no responde a las reglas y contenidos definidos por el profesorado, y tampoco es el territorio propio del mundo del alumnado, pero incorpora contenidos y metas de ambos universos en un espacio de diálogo en el que los diversos significados no son censurados ni estigmatizados. El tercer espacio se proyecta desde una perspectiva creativa y dialógica: creativa en el sentido en que se entiende la imaginación y la creación personal como proceso fundamental del desarrollo personal (Connery, JohnSteiner y Marjanovic-Shane, 2010; Vygotski, 2007) y dialógica en el sentido de que supone un encuentro de subjetividades en igualdad de derechos (Bajtín, 2000; Matusov, Smith, Soslau, Marjanovic-Shane, y von Duyke, 2016). Como señala Rebollo (2006), es en este diálogo creativo donde se generan prácticas discursivas en las que se desarrollan formas de pensamiento cualitativamente distintas y el metaconocimiento sobre cuándo y cómo es apropiado utilizarlas para mediar en los conflictos a través de la escucha.

En el tercer espacio surge la posibilidad de legitimar la voz de las personas participantes, de manera que facilita la negociación de significados y la construcción de metas compartidas, lo que permite la apropiación del sentido para el conjunto de participantes. Las prácticas educativas basadas en FdI nos aproximan a las prácticas de los y las aprendices respecto a los contextos de actividad y vida para conectar sus motivos. Así pues, al poner a los y las estudiantes en el centro, y basarse en la interpelación de su voz personal y en la posibilidad de transformar la práctica educativa, se establecen vínculos 
entre la práctica comunitaria y la escolar, y pueden darse los fenómenos de legitimación y de transformación característicos del tercer espacio.

A continuación vamos a presentar una experiencia de intervención educativa mediante FdI con chicos y chicas entre 12 y 14 años de una escuela que ha sufrido procesos de segregación y guetización. Pretendemos describir y analizar los procesos de diálogo promovidos desde los FdI y la creación de terceros espacios gracias a la legitimación de los referentes propios del mundo del alumnado, y que dotan de sentido a la experiencia escolar.

\section{CONTEXTO. EL INSTITUTO-ESCUELA EL TIL·LER}

Nuestro análisis se desarrolla en el marco de un proyecto de investigación acción que a lo largo de 20 años ha supuesto la intervención real en distintos contextos educativos formales y no formales (Lalueza, Sánchez-Busqués y García-Romero, 2019), el Proyecto Shere Rom, que supone la colaboración de investigadores, maestros, estudiantes universitarios en prácticas de Aprendizaje-Servicio y activistas, junto con niños y niñas en contextos de exclusión social. Este proyecto se basa en los principios de la Quinta Dimensión, un modelo de comunidades de prácticas que se desarrolla mediante la colaboración interinstitucional (universidad, escuelas y entidades locales), intergeneracional (niños, estudiantes universitarios y educadores) e intercultural (la cultura hegemónica del sistema educativo y las de referencia familiar y comunitaria de los participantes), mediante un conjunto de actividades dirigidas a metas, basadas en el aprendizaje colaborativo y enmarcadas en un sistema de significados compartidos (Nilson y Nocon, 2005).

Concretamente, la experiencia que vamos a presentar aquí se está llevando a cabo en el Instituto-Escuela El Til·ler, situado en el Bon Pastor, un barrio del área de acción social prioritaria del río Besòs en la ciudad de Barcelona. Ha funcionado durante más de cuatro décadas como escuela de enseñanza infantil y primaria (3 a 12 años), con un fuerte componente de alumnado gitano. Con la llegada de la inmigración a partir de los años 90 del pasado siglo, la composición étnica del centro sufrió un cambio dramático, ya que el único alumnado autóctono es el gitano, al 50\% con alumnado inmigrante. A pesar de situarse en un contexto social de riesgo, la orientación hacia la inclusión de esta escuela ha mantenido durante todos estos años unos muy bajos niveles de absentismo en primaria. No era así en el centro de secundaria de la misma zona, de manera que el instituto correspondiente se mantenía con una escasa población, con un abandono creciente a medida que se avanzaban los cursos (de los 12 a los 16 años) y con muy malas expectativas de éxito para su alumnado (una bajísima ratio de graduados en secundaria en relación a la entrada en primer curso). Es por ello que las autoridades educativas decidieron convertir la escuela de primaria en un instituto-escuela (3 a 16 años), aprovechando los vínculos establecidos entre el centro y las familias y así afianzar la continuidad entre primaria y secundaria.

Desde 2017 se ha transformado en un nuevo centro, con una enseñanza basada en proyectos, en el trabajo colaborativo y en un gran número de alianzas con entidades del entorno. Desde la universidad hemos acompañado al centro en esta transformación, con la participación de investigadores y estudiantes, y colaborando con las maestras así como con educadores gitanos de entidades comunitarias. El proyecto de nuestro equipo consiste en el desarrollo de comunidades de práctica en primaria y secundaria, siguiendo el modelo Quinta Dimensión y en el último año la incorporación de proyectos de FdC en preescolar y FdI en secundaria. 


\section{MÉTODO: INVESTIGACIÓN POR DISEÑO DE PRÁCTICAS EDUCATIVAS CON FONDOS DE IDENTIDAD}

Enmarcado en un proyecto de investigación acción, este estudio es un análisis del funcionamiento de una comunidad de prácticas (Wenger, 1999) cuyo diseño se ha elaborado mediante la colaboración entre un equipo de investigación de la Universitat Autònoma de Barcelona y el equipo docente del Instituto-Escuela El Til-ler. Podemos considerarlo una investigación por diseño (Cole, 2016; Engeström, 2011; Gutiérrez y Vossoughy, 2010), en tanto que el fenómeno investigado es el mismo diseño y ejecución de la intervención, así como los fenómenos interpersonales e identitarios que emergen en el curso de su devenir. El análisis de eventos de creación de significado (Bajtín, 2000) nos permitirá identificar los elementos de la práctica educativa que fomentan la emergencia de un tercer espacio, así como los procesos de aprendizaje expansivo (Engeström, 2011).

\section{DESCRIPCIÓN DE LA INTERVENCIÓN}

El proyecto trata de poner en marcha un proceso educativo congruente con las necesidades de aprendizaje del alumnado así como de sus conocimientos, habilidades y destrezas emanadas de sus recursos y prácticas culturales. Para hacerlo, se ha trabajado a partir del marco teórico-metodológico de los Fondos de Identidad, es decir, con aquellos artefactos, tecnologías o recursos históricamente acumulados, culturalmente desarrollados y socialmente distribuidos y transmitidos, esenciales para la autodefinición, autoexpresión y autocomprensión de los alumnos. La finalidad es poder generar aprendizajes significativos que puedan producir continuidades entre las prácticas y recursos culturales de los diferentes contextos de vida del alumnado (Subero, Brito y Esteban-Guitart, 2017).

Aunque la intervención del proyecto se prolonga a lo largo de todo el curso, este estudio se circunscribe a una propuesta de FdI consistente en 7 sesiones de 2 horas con periodicidad semanal en dos grupos de ESO (Figura 1).

\begin{tabular}{|c|c|c|c|c|c|c|c|}
\hline $\mathbf{N}^{0}$ de sesión & 1 & 2 & 3 & 4 & 5 & 6 & 7 \\
\hline $\begin{array}{l}\text { Actividades } \\
\text { realizadas }\end{array}$ & $\begin{array}{l}\text { Presentación } \\
\text { y creación } \\
\text { del círculo } \\
\text { significativo }\end{array}$ & $\begin{array}{c}\text { Actividades } \\
\text { para tratar las } \\
\text { prácticas } \\
\text { identitarias }\end{array}$ & $\begin{array}{l}\text { Actividades } \\
\text { para tratar los } \\
\text { fondos } \\
\text { sociales de } \\
\text { identidad }\end{array}$ & $\begin{array}{l}\text { Actividades } \\
\text { para tratar los } \\
\text { fondos } \\
\text { culturales, } \\
\text { geográficos e } \\
\text { institucionales } \\
\text { de identidad }\end{array}$ & \multicolumn{3}{|c|}{$\begin{array}{c}\text { Creación del } \\
\text { libro de } \\
\text { artista }\end{array}$} \\
\hline
\end{tabular}

Figura 1. Cronograma de la intervención 
En la primera sesión se ha realizado el círculo significativo, una de las técnicas propuestas en la Multi-metodología Autobiográfica Extendida (MAE) (Esteban-Guitart, 2012). La tarea es individual y consiste en dibujar un círculo y situar dentro de él los objetos, actividades, personas, instituciones, aficiones, elementos culturales, etc. más importantes de los alumnos y las alumnas, de las cuales las que aparecen más centradas en la imagen son las más importantes para la persona. La realización de esta actividad ha permitido ahondar y reflexionar entorno a las diferentes maneras en las que se expresan los fondos de identidad (Esteban-Guitart \& Moll, 2014) y, por lo tanto, conduce y sirve de nexo en las diferentes sesiones posteriores.

En las siguientes tres sesiones se ha trabajado sobre los diferentes fondos de identidad. En cada sesión se ha dado la oportunidad al alumnado de decidir cómo querían trabajar: a través del arte plástico, de la representación teatral o de actividades lingüísticas. Basándonos en la perspectiva de Moreno (2016) planteamos un método de intervención socioeducativa en el que el arte, en sus diferentes disciplinas facilita un acceso a la cultura, a una ruptura de mirada estereotipada, al desarrollo de la resiliencia y los procesos de simbolización. En los procesos artísticos, la identidad se materializa como una muestra de vida, con escenas, personajes, objetivos y temas que en términos narrativos nos permiten conferir intencionalidad, integrar los sucesos y dotar de unidad y propósito nuestras vidas (Bruner, 1997). La diversificación de las áreas de expresión, a través de la narrativa escrita, de la expresión corporal o teatral y la expresión gráfico-plástica, da la posibilidad de escoger la disciplina con la que sienten mayor afinidad, legitima diferentes formas de expresión y las pone todas al mismo nivel.

La estructura propuesta para cada una de estas tres sesiones fue la siguiente:

- Inicio: en grupo aula, presentación del tema del día para buscar la motivación del alumnado.

- Desarrollo: actividades en grupos divididos en los tres espacios de participación (arte plástico, representación teatral o actividades lingüísticas). Dichos grupos son conformados según sus preferencias en cada una de las sesiones, de manera que cada alumno se apunta para el siguiente día a uno de los tres espacios.

- Cierre: reflexión en grupo aula, revisando círculo significativo para decidir si se quiere añadir, eliminar o mover algún elemento; introducir próxima sesión, apuntarse al próximo taller y despedida.

Las últimas tres sesiones se han dedicado a la creación del libro de artista (Figura 2), un libro-folleto individual de seis páginas para recoger y plasmar todo aquello que hemos ido trabajando en las sesiones. Para su elaboración se ofrecen múltiples opciones de representación, de manera que continúa sosteniendo la filosofía que ha vertebrado la base del proyecto basada en la libertad de elección para el alumnado, a través de actividades en las que se puedan escoger y explorar diferentes técnicas expresivas y creativas.

Este formato de producción permite cerrar el proceso para recoger los principales aspectos que han surgido durante las sesiones, reificando y legitimando así su propia voz. Con ello se visibiliza lo que cada cual ha considerado importante para sí mismo después de la exploración. Desde el punto de partida de una creación para "contar nuestra historia o contarnos a nosotros mismos" se emplean materiales y técnicas como el collage, el dibujo, el cómic o la pintura, entre otros. 


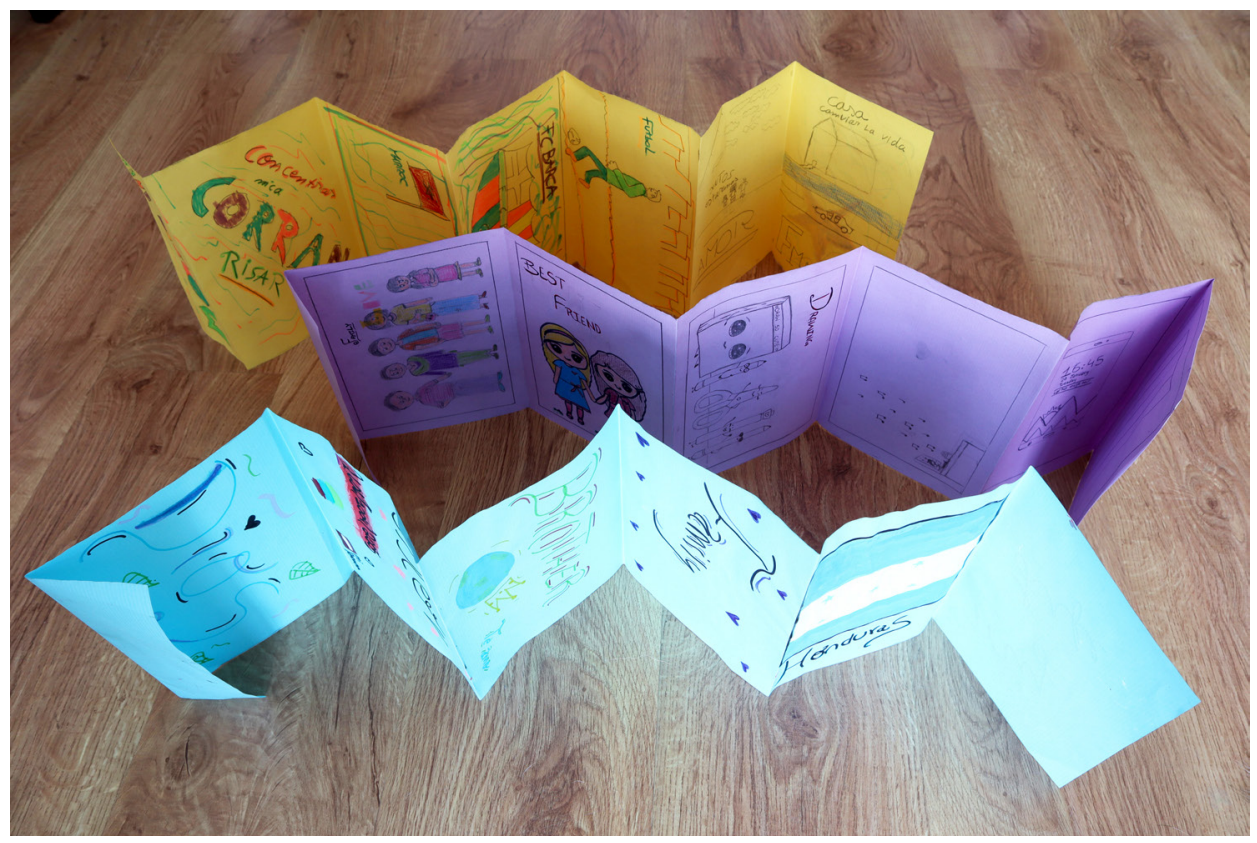

Figura 2. Ejemplos de libro de artista

\section{PARTICIPANTES Y GENERACIÓN DE INFORMACIÓN}

Las actividades se llevaron a cabo en dos clases paralelamente de primero y segundo curso de Enseñanza Secundaria Obligatoria (ESO). En primero participaron 1 profesora, 25 alumnos y alumnas y 3 investigadoras, mientras que en segundo participaron 2 profesoras, 18 alumnos y alumnas y 4 investigadoras. Algunas de ellas iremos apareciendo en el texto de bajo con pseudónimos.

Esta información es generada y registrada a través de diferentes instrumentos y en diferentes formatos. En este sentido, la MAE que guía el proceso de intervención se refleja también en la forma de la información a analizar:

a) Los diferentes productos elaborados por los alumnos suponen en sí mismos material de análisis sobre aquello que han considerado importante plasmar $\mathrm{y}$, por lo tanto, es relevante considerarlos en conjunto con los datos sobre el proceso educativo.

b) Las investigadoras llevamos a cabo una observación participante, cumplimentando notas de campo con un registro riguroso en el que se incluyen interacciones verbales y no verbales explícitas en la actividad. Permiten también la reflexividad (Hernández, Sancho \& Creus, 2011; Willig, 2013) profundizando en el sentido de la actividad y al mismo tiempo hacer a las investigadoras conscientes de su posición situada.

c) Contrastamos las notas de campo con los registros de las discusiones con otros dos 
agentes: Las reuniones de planificación y valoración con las profesoras responsables de la actividad, y las reuniones del equipo de investigación que incluye a investigadores no participantes en la práctica concreta pero sí implicados en el proceso de diseño y evaluación. El diálogo con las profesoras otorga una perspectiva diferente sobre lo ocurrido, y con otros investigadores fomenta un extrañamiento de la actividad y permite la una doble perspectiva ética y émica.

Los datos aparecerán en el análisis marcando tanto la voz del hablante como el tipo de documento de procedencia. Respecto a las notas procedentes de la observación participante, dado que han sido escritas en un documento colaborativo y queremos enfatizar la voz que habla y no la que lo registra, no detallamos qué investigadora concretamente escribió cada fragmento.

\section{ANÁLISIS DEL TERCER ESPACIO Y FONDOS DE IDENTIDAD}

En primer lugar, es importante mencionar que nuestra investigación se encuentra en una fase inicial, y que no hemos tenido la voluntad de un análisis sistemático y exhaustivo de todos los datos producidos, lo cual encaja dentro de la perspectiva de una investigación dialógica (Matusov, Marjanovic-Shane y Gradovski, 2019) que basa su validez de análisis más en la dialogicidad con participantes y audiencia (fomentando la transparencia del análisis) que en la exhaustividad y sistematización. Aquello que nos interesa en este momento es atender a los eventos de creación de significado (Bajtín, 2000) que emergen de las prácticas y que nos pueden dar información sobre los sentimientos de legitimación para tratar temas personales que permitan trazar rumbo a transformaciones futuras. Por ello, elaboraremos un análisis narrativo de eventos que consideramos significativos tanto por la apertura de procesos de diálogo como por la relevancia del contenido de estos.

Contrastaremos este análisis con las características de la actividad en cada evento emergente, de manera que podamos vislumbrar qué elementos de la práctica educativa de FdI caracterizan y hacen posible el tercer espacio. Así, nos aproximamos al análisis desde una perspectiva inductiva, buscando indicadores que nos hablen de forma fundamentada sobre los procesos de legitimación y de diálogo en esta práctica educativa, que buscaremos tanto en la propia voz de los alumnos como en la observación de las investigadoras y maestras.

\section{RESULTADOS Y DISCUSIÓN}

\section{APROXIMACIÓN GENERAL}

Con la intervención intentábamos generar un espacio donde los alumnos desarrollasen narrativas identitarias, que se vieran legitimados para tratar temas de su propio interés, y con la posibilidad de establecer un entorno de diálogo o tercer espacio.

La reacción de los alumnos en los primeros momentos fue sorpresa e incluso de resistencia a nuestra propuesta. Numerosas expresiones mostraron que tratar sus temas personales les resultó novedoso e inapropiado. 
Da igual lo que digamos, si vais a hacer lo que queríais.

(Eduardo_NotasCampo_05.02)

Lo que me sorprendió fue que porque me interesara por lo que pensaban, dieran por hecho que era psicóloga. Creo que eso es un ejemplo de lo poco que son escuchados estos chicos en su actividad cotidiana. (Alicia_ReuniónInvestigación_26.02)

Estas menciones a lo inhabitual que es tratar su vida y sus preocupaciones en el instituto, sugiere que el posterior tratamiento de forma explícita de temas personales puede ser un indicador de que se sienten legitimadas para hablar con voz propia. Los eventos han sido numerosos y variados en su forma y temas: relaciones paterno-filiales, la muerte de personas importantes, la experiencia de migrar, las diferencias entre cultura propia y escolar, desahucios, racismo, etc.

Del total de 48 alumnos y alumnas que han participado en este proyecto, 35 de ellos realizaron la autoevaluación el último día de sesión. De estos, 27 comentaron que les ha gustado el proyecto y les ha parecido muy creativo. Algunos de los motivos que dan son:

Me parece original y creativo porque como es personal puede interesar a cualquier persona te guste lo que te guste. (Aitana_Autoevaluación_25.02)

Es algo que nunca había hecho y me ha gustado; cada cosa era diferente y chula. (Diego_Autoevaluación_25.02)

Por otro lado, al preguntarles sobre si realizar estas actividades les ha ayudado a descubrir alguna cosa que no sabían de ellas mismas, hay diversidad de opiniones:

He descubierto que hay un grupo de 5 personas que eran importantes para mí. Es algo que supongo que ya sabía pero gracias a esto ahora veo que me importan mucho más de lo que yo pensaba. (Julia_Autoevaluación_25.02)

Cuando me paré a pensar me sorprendí a mí misma porque hay muchas cosas que no sabía que me gustaban. (Samara_Autoevaluación_25.02)

Julia hace referencia a un "redescubrimiento", a haber hecho relevancia a algo que no le resulta nuevo pero que le gusta haber visto proyectado, mientras que Samara directamente dice que ha descubierto cosas que no sabía de sí misma, lo que destaca el potencial de la actividad para el autodescubrimiento. Algunos han comentado que la actividad del libro de artista es de sus favoritos, pero otros dicen que las actividades de crear en general porque les gusta dibujar, otros las de actuar y otros las de comunicar. Uno de los chicos comenta que lo que más le ha gustado ha sido poderse dividir en grupos. De hecho, en general al preguntar al grupo clase todas han dicho que preferían hacer la actividad tal como la hemos hecho, separados por grupos y escogiendo en qué espacio querían estar. Estos dos elementos: el elegir el espacio en que quieren participar y el dividirse en grupos fomenta un clima de confianza necesario para entrar en un diálogo auténtico.

\section{ANÁLISIS DE EVENTOS}

No estamos en disposición, en este momento de nuestra investigación, de realizar un análisis sistemático y exhaustivo, pero sí creemos fundamental el aproximarnos a algunos 
eventos concretos para poder explorar un primer nivel de análisis, por lo que exponemos a continuación algunas de estas experiencias concretas que se han producido en la práctica educativa. Concretamente, expondremos cuatro eventos: Mi vida a los 12 años, Descubrimiento de la cultura, Asimilación y resistencia e Identidades en diálogo. Estos eventos giran en torno a dos ejes principales: el primero de ellos sobre el racismo y la violencia, y el segundo de ellos sobre cultura e identidad.

\section{EVENTO 1. MI VIDA A LOS 12 AÑOS}

Durante las sesiones para elaborar el libro de artista, el alumnado empieza por elaborar un esbozo recuperando aquellas cosas más importantes o significativas que quieran plasmar en la creación y posteriormente pasan a elaborarlo sobre el libro-folleto. "Mi vida a los 12 años" es el título del libro de artista de Víctor, quien en un inicio expresaba que no iba a hacer el libro porque él no hacía nada, lo cual manifiesta explícitamente y en voz alta varias veces con convicción.

Me acerco a él y le pregunto cómo lo lleva, a lo que él responde de nuevo "es que no sé qué poner, no hago nada”. Ante su respuesta, le respondo "¿cómo que no? Sí que haces cosas. Ahora mismo estás haciendo cosas, venga vamos a ver qué cosas haces”. (Yue_NotasCampo_11.02)

A partir de aquí, Yue y Víctor empiezan a entablar un diálogo del cual emergen diferentes temas vinculados a la escuela y la familia. En esta, el chico manifiesta diferentes situaciones en las que se refiere a la violencia.

Víctor: ¿Que qué hago? Pegar. A parte de pegar, no hago nada más

Yue: Hombre, pegar es hacer algo

Víctor: Sí pero no voy a poner pegar aquí

Yue: ¿Por qué no? Si tú crees que pegar forma parte de ti, puedes ponerlo. Pero a ver, ¿por qué pegas exactamente?

(Víctor y Yue_NotasCampo_11.02)

En esta conversación, la investigadora/educadora ofrece un espacio para expresarse abiertamente, legitima su voz y muestra interés por él. Aunque no sea algo socialmente aceptado, la exploración de la violencia como algo propio de Víctor es importante para Yue, pues es necesario legitimar totalmente la voz de las participantes de las prácticas de FdI, no solamente cuando su mensaje es socialmente aceptable. Se marca así la importante diferencia entre que sea legítimo hablar de todo, aunque no todo mensaje vaya a ser aceptable, sino precisamente discutido. En el diálogo, son las voces las que están en igualdad de derecho, no los mensajes (Bajtín, 2000). De hecho, este no es un tema nuevo, sino que emergió en dos sesiones anteriores. En la primera, mediante una actividad de expresión teatral, realizó junto a dos compañeros más situaciones de acoso escolar violentas. En otra sesión, en una actividad de expresión artístico, dijo "Si pudiera dibujar algo con muchas personas ¿sabes qué sería? A mí matando a un montón de gente de este colegio" (Víctor_NotasCampo_28.01). Por lo tanto, Yue insiste en mostrar a Víctor que todo aquello que él considera que es o hace, es un tema de conversación a explorar y problematizar, buscar motivos e implicaciones. 
Víctor explica que como a él le pegan o le han pegado, él también lo hace. Al preguntarle si sus hermanos también le pegan, dice que no pero que son muy pesados. Intento ahondar en esto de la violencia que él recibe, y me dice que le pasa muy a menudo pero que él se lo toma a broma y con humor, que él es así y le gusta mucho hacer bromas, que cuando le pasa en el cole él intenta pasar con humor. Después de explicarme esto, le sugiero que quizás el humor sea algo característico de él y que podría añadirlo en el libro si quisiera. Le parece bien pero me dice "pero, y cómo lo hago? dibujo como que me están insultando y me pongo yo riendo?" "Sí, así mismo está bien, no? Si crees que es algo que te define creo que es buena idea”. (Yue_NotasCampo_11.02)

A través de la posibilidad de hablar de un tema que en principio Víctor percibía como ilegítimo (una parte de él claramente no aceptada) este encuentra otra característica propia que explorar, el humor. Al mismo tiempo, aunque no en la conversación, sí ha estado reflexionando sobre más elementos propios.

Cuando coge su hoja de esbozo, veo que ya tenía anotadas algunas cosas que le gustaba hacer: los deportes (básquet y fútbol) y las matemáticas. Mientras está haciendo el esbozo sobre el humor, hablamos sobre estas cosas que le gustan. En un momento dado está Mónica (tutora y profesora de matemáticas) pasando cerca y nos escucha. "¿Qué ha dicho de las mates?” Le respondo "dice Víctor que le gustan las mates". Mónica está sorprendida y le pregunta a él directamente, incrédula “ ¿A sí, Víctor? ¿Te gustan las mates?” Y Víctor le responde que sí, tímidamente. Por su reacción, parece ser que Mónica no lo sabía. Me llama la atención cómo le responde Víctor, ya que cuando me lo explicaba a mí no se mostraba para nada tímido ni reservado. (Yue_NotasCampo_11.02)

La negativa inicial de Víctor a hablar de sí mismo no tiene que ver con una falta de consciencia o intereses, pues sí los ha explorado de forma plástica, sino que más bien tiene que ver con una percepción de que no son temas de conversación relevantes, y es a través de la actividad creativa y de la interpelación constante de Yue que finalmente encuentra un espacio que no existe en el aula ordinaria. Testigo de esto es la sorpresa de Mónica sobre que a Víctor le guste esta disciplina, a la vez que la renovada vergüenza de él a hablar de esto con la profesora. Esta vergüenza puede ser entendida como una precaución en una situación en la que aquello que dice no es tomado en serio y escuchado atentamente, sino evaluado.

Víctor narra otro tipo de situaciones en las que no es tomado en serio en el momento en el que él y Yue vuelven sobre el tema de la violencia:

Le pregunto si alguna vez ha probado de hacer alguna cosa distinta en vez de pegar o reírse como respuesta. Rápidamente responde "Sí claro, decírselo a mis padres. Pero no funciona porque no me creen" [...]Le pido si me puede explicar más esto para entender a qué se refiere y me hace un paralelismo con el cuento del lobo y las ovejas: "Alguna vez le he dicho en broma a mis padres que por ejemplo mis hermanos se están peleando, y cuando pasa de verdad pues ya no me creen”. Cuando me explica esto, le digo que lo puedo llegar a entender y le sugiero que podría explicar esto, ya que me dice que es algo que le ocurre con frecuencia. [...] le comento que quizás lo podría 
explicar así como me lo había explicado a través de viñetas. Parece ser que enseguida lo capta y se dispone a dibujarlo. Al terminar me lo enseña y veo que en la última viñeta se ha dibujado a sí mismo en medio de los dos hermanos que se están peleando. Le felicito porque se entiende muy bien cómo lo ha narrado y le pregunto si al final es él que tiene que resolver la situación. "Claro, siempre me toca a mí arreglarlo" (Yue_NotasCampo_11.02)

Por una parte, Víctor refleja en su historia cómo siente que no es tomado en serio por diferentes personas de su entorno. Al mismo tiempo, es a través de la narración que de nuevo descubre otro elemento de sí mismo que emerge como relevante: al que le toca arreglar las cosas. Esta exploración es facilitada por Yue, que de nuevo recoge sus propios descubrimientos y se los muestra.

Le verbalizo que sí sabe hacer cosas, mientras le enseño todo lo que ha hecho en el esbozo. En la misma sesión se dispone a pasarlo en limpio y al final de la sesión prácticamente ya lo ha terminado. (Yue_NotasCampo_11.02)

Esto señala la importancia de los actos de reconocimiento (Taylor, 1996) en la legitimación de la voz de Víctor. Podemos ver también cómo la referencia a este momento sirve para trasladar un acto de reconocimiento a otro alumno.

La semana siguiente hablamos de ello con Javi y Toni. Víctor terminó enseguida el libro y nos pusimos a ayudar a Javi, quien no había asistido la sesión anterior y no sabía qué hacer, similar a Víctor. Aproveché para hacerle ver que Víctor tampoco sabía qué hacer. (Yue_NotasCampo_11.02)

Javi y Víctor comparten la misma visión sobre lo que la escuela espera de ellos, su aseveración de que no saben hacer nada se basa en que han aprendido que no saben hacer nada según lo que el modelo normativo escolar espera que hagan (Sebastián, Gallardo y Calderón, 2016), pues las actividades educativas están centradas en el currículum y no en sus personas (Matusov et al., 2016). La actividad basada en FdI las interpela directamente y permite hablar de valores personales y sociales, lo cual no deja de ser arriesgado, como vemos en el siguiente párrafo.

Al hacer alusión al tema que trató Víctor, los otros dijeron compartir la violencia en su forma de relacionarse. El tema transcurrió hacia la pornografía (como algo que le gustaba a Javi), y pasamos a discutir sobre el machismo y el racismo a partir de comentarios que hicieron cuando hablaban, tales como "a X le gustan las pollas negras". Cuando les hice notar que esta expresión era ofensiva, se rieron y se reafirmaron en que no lo era, volviendo a decir lo mismo. Hacia el final de la sesión estaban los tres junto a otros chicos charlando. En un momento Javi recriminó a un compañero un comportamiento llamándole "moro" y José, otro compañero, le hizo notar que yo lo había escuchado. Javi se reafirmó diciendo que le daba igual ya que no era ofensivo, porque lo hacía para corregir un mal comportamiento. Víctor le explicó con una sonrisa que, aunque para algo bueno, esa palabra en sí mismo era racista, y por lo tanto ofensivo. Javi se quedó quieto pensando, le devolvió la sonrisa y le dio la razón. (Yue_NotasCampo_11.02) 
Yue entra al diálogo sin imponer su código moral, sino argumentando su perspectiva en pie de igualdad. Esto resulta seguramente difícil en el momento educativo, al temer que se reafirmen actitudes de discriminación e imaginarios racistas que parecen normales inicialmente para estos chicos. Sin embargo, la combinación de interpelar a los estudiantes sobre temas éticos como el racismo, junto con la falta de imposición de normas morales, es la que finalmente permite que discutan y construyan códigos morales propios. En este evento hemos visto cómo Víctor admite utilizar la violencia de forma naturalizada, pero permite también hablar de las razones de esto y explorar sus propias estrategias para evitarla (humor y mediación), de la misma forma que muestran un imaginario racista, al tiempo que sacarlo a la luz les permite empezar a cuestionarlo.

\section{EVENTO 2. DESCUBRIMIENTO DE LA CULTURA}

Para tratar sobre los fondos culturales de identidad en el espacio dedicado a la expresión lingüística proponemos una actividad en la que a partir de unas fotografías sobre personas diversas (con distintos aspectos, de distintas culturas, etc.) conversamos sobre cuál creen que es la cultura de cada una de ellas y cuál creen que es su historia. En esta actividad podemos ver cómo el alumnado se aproxima de una forma periférica a crear narrativas basadas en la cultura. Ante esta inexperiencia, el marco conceptual que toman como referente parece basado en el racismo, conteniendo los tres elementos que Buraschi y Aguilar-Idáñez (2017) manifiestan como característicos de este la diferenciación, la esencialización y la estigmatización. Un ejemplo de ello es cuando trataron de inventarse la historia de un chico joven negro y no consiguieron pensar nada positivo de su historia:

Este chico negro debe ser muy pobre y cuando era pequeño y vivía en su país debió de pasar mucha hambre porque en su casa no tenían dinero. Sí, y seguro que se metían con él por ser negro. (Miguel_NotasCampo_04.02)

La referencia a la persona negra lleva a Miguel de forma muy directa a pensar en una forma de vida estigmatizada, vinculada a la pobreza y en la migración forzada. La misma estigmatización se da al trazar un modelo adulto, pero en este caso se pasa de la pobreza y la vulnerabilidad a la delincuencia, como vemos en el comentario de José respecto a la historia de un hombre negro:

Este es fácil, seguro que es narcotraficante. (José_NotasCampo_04.02)

En esta actividad, ante la proyección de personas diversas, el factor de racialización se presenta como un elemento diferenciador fundamental para el alumnado, que además coincide en relatar historias de marginalización a dos personas diferentes, lo cual nos lleva a una posible esencialización, ya que la marginalidad aparece naturalmente ligada al hecho racial.

Por otro lado, al hablar e hipotetizar sobre las culturas de dichas personas se vio cómo solamente vinculan cultura a religión, pensando entonces que aquellas personas que no creían en ningún Dios no tenían cultura alguna. Este debate desembocó naturalmente en hablar de su propia experiencia, dando lugar a la siguiente conversación: 
José: Yo no tengo ninguna cultura, no creo en Dios ni voy a la iglesia.

Miguel: Sí, yo en cambio soy gitano y creo en Jesús, aunque no vaya al culto porque Jesús está siempre allí. (Miguel y José_NotasCampo_04.02)

Esta referencia es tomada por las investigadoras/educadoras, que mencionamos otros elementos culturales, para ampliarlo más allá de lo religioso. La conversación transcurre alrededor de diferentes características y prácticas culturales de diferentes personas presentes. Se empieza así a perfilar el hecho de que todas tenemos una cultura, y Miguel y José llegan a la conclusión de que el segundo es "payo", y la paya es una cultura en sí misma. La conversación transita a reflexionar sobre qué cosas diferentes tiene la cultura gitana (la de Miguel) de la paya (de José). Miguel le explica a José en qué consiste el rito del pañuelo y le explica también que para su cultura la familia es lo más importante. Ante estas explicaciones y después de un rato de hablar sobre cultura gitana:

José le dice que por la calle se comenta que los chicos gitanos pueden tener relaciones sexuales con quien quieran pero las chicas no porque le hacen el rito. Yo les comento que el año pasado me explicaron que el rito del pañuelo tenía sentido para evitar que nadie de la familia (entendida como linaje) pudiese abusar de las niñas porque si lo hacía se mancillaba toda la familia y por tanto también a quien hubiera abusado de ella. (Izar_NotasCampo_04.02)

Ante la sugerencia de Izar, Miguel empieza a complejizar aquello que daba por natural de su propia cultura y a plantearse sentidos diferentes a los conocidos. A través de esta conversación, la noción de cultura se complejiza para ambos, para José deja de ser aquello que tienen las otras personas, a ser algo que le atraviesa también a él, mientras que, a través del diálogo, Miguel se extraña de lo que es natural para él y complejiza los significados. Esta problematización o complejización contrasta con la disposición inicial a la esencialización o estigmatización de los rasgos racializados.

En este mismo evento se da un episodio diferente. En el momento en que planteamos unir todas aquellas cosas importantes y hablar especialmente de los fondos geográficos de identidad, diseñamos cómo sería su barrio ideal (qué personas vivirían en él, dónde estaría situado, qué cosas tendría, cómo sería su casa, etc.) y tras haberlo dibujado miramos sus círculos significativos para ver si aparecía todo lo que ellos habían pensado como importante. A partir de aquí hacen algunos cambios. Como es el caso de José, que al plantearse con quién viviría vuelve sobre su círculo y lo corrige. Su mismo tono nos permite interpretar una reivindicación a sí mismo, al haber olvidado alguien importante:

No me he acordado de poner a mi tía que tiene Síndrome de Down. Para mí es súper importante y quiero tenerla cerca siempre. Así que ella viviría dentro de mi casa, conmigo. (José_NotasCampo_04.02)

A continuación Miguel comenta que a pesar de que para él es muy importante Barcelona (donde están sus amigos y familia), le gustaría que su barrio fuese en Uruguay, donde viven sus abuelos. Al hablar sobre la importancia de su familia explica que quiere que vivan en el mismo barrio aunque no en la misma casa y le explica a José que aunque haya puesto la 
religión como algo importante en su círculo no quiere ninguna iglesia en su barrio porque el Jesús en el que cree está en todas partes. Después de esto, se empieza a imaginar cómo querría su casa: con vistas al mar, con una pared de cristal, etc. y le pregunta a José cómo le gustaría a él que fuera la casa, puesto que en el círculo significativo de José aparece como uno de los elementos importantes "Casa".

Me da igual qué casa sea, quiero una casa grande donde quepa yo, mi tía y Miguel. Lo he puesto en mi círculo porque tener una casa donde vivir es una de las cosas más importantes que hay. (José_NotasCampo_04.02)

José está aquí haciendo una clara alusión a sus necesidades y prioridades actuales. La experiencia de haber sufrido un desahucio y tener que vivir con otra familia crean una clara diferencia en qué es lo importante sobre tener una casa: mientras que uno piensa en cómo es su ideal, el otro se queda simplemente en la unidad urgente de tener una para él, enunciando esto como una de las "cosas más importantes que hay". A través de la actividad enuncia principios morales o sociales descubiertos en su experiencia reciente. Dicha conversación y la posibilidad de tratar temas culturales y de entrar en diálogo real sobre cosas que no habían tenido oportunidad de hablar apareció en el marco de un grupo muy reducido de alumnos, constatando una vez más que para que el espacio sea seguro y no se sientan expuestos necesitan de grupos reducidos en los que se crea un espacio de mayor intimidad.

\section{EVENTO 3. ASIMILACIÓN Y RESISTENCIA}

En el espacio de creación plástica sobre fondos sociales comenzamos con una dinámica en la que las cuatro asistentes se dibujaban entre sí. Esta actividad dio pie a diversos conflictos entre ellos relacionados con el aspecto físico. Alicia, tratando de mediar la situación, preguntó por la actitud agresiva que mostraban entre ellos, hablándose con insultos y con cierta violencia.

Abdu dijo "aquí si no eres así te toman el pelo". Le pregunté si le había pasado en otras ocasiones y hablamos sobre otras experiencias en las que se habían sentido amenazadas. "Es que es lo que hay" Dijo Fatiha, a lo que Abdu añadió: "Si no, no te respetan. Ya me ha pasado muchas veces y aprendí a ser así”. Me explicaban cómo los conflictos son mediados entre ellas bajo amenazas. Al preguntarles por separado si querían hablar sobre lo que había pasado contestaron que no "porque si no nos buscamos problemas fuera” (Alicia_NotasCampo_24.01)

En esta interacción, Fatiha y Abdu dejan claras las normas que han aprendido como propias de su entorno, y que tienen que ver con el uso de la violencia o el poder como fuerza mediadora de las relaciones. Sin embargo, se adhieren a estas normas por supervivencia, no por una decisión propia genuina. Esto es interpretable como propio de contextos en exclusión o en riesgo, atravesados por diferentes violencias estructurales relacionados con dinámicas de segregación y marginalización. Lo que vemos, por lo tanto, es que dentro del instituto no hay modelos alternativos de relación sino que se convierte una extensión del "fuera" al que Abdu hace referencia. 
Abdu habló sobre su paso por el centro educativo, destacando experiencias negativas que había vivido hasta mostrar rechazo sobre las personas inmigrantes y gitanas. Se reafirmaba como autóctono y se situaba "en el otro lado", ser él quien rechaza:

Abdu: No quiero estar en esta escuela de gitanos y gentuza de otros países.

Youseff: Pero si tú también eres de otro país.

Abdu: ¡No! ;Yo soy de aquí, yo he nacido aquí!

(Abdu y Youseff_NotasCampo_24.01)

En esta situación, el estudiante expresaba enfadado sus dificultades relacionales en el colegio y destacaba lo positivas que eran las relaciones con amigos que tenía en su colegio anterior. El chico desarrolla así una resistencia a la estigmatización que sufre y que identifica con el centro y grupos minorizados que asisten. Intenta diferenciarse de esta a través de una estrategia asimiladora (Berry, 2001) que acarrea un concepto estático de cultura. Su opción de asimilación y diferenciación necesita de una barrera clara que proteja la alteridad entre los migrados y él, de tal manera que quede en el lado no estigmatizado y, por consecuencia, reproduce la misma violencia.

La sesión continuó y Abdu se centró en un dibujo en el que aparecía él jugando al fútbol con sus amigos del colegio anterior. En la expresión plástica encuentra una forma de expresión en la que se ve valorado y reconocido por las educadoras.

El dibujo permitió a otra de las alumnas del grupo, Sara, hacer referencia a personas de su familia. A través de su propia creación conectó con recuerdos y experiencias concretas:

Yo le dije que parecía concentrada y entonces respondió que dibujar le recordaba a su abuelo. "Ojalá dibujara como él... Su casa está llena de cuadros nuestros que él ha hecho. De mí, de mis primos... iEstamos todos! Apenas le caben. Pero ahora ha tenido que quitar muchos. Y bueno, ya no puede dibujar por desgracia, ya no está muy bien" (Alicia_NotasCampo_24.01)

Fatiha, por otro lado, representó en su trabajo a artistas que eran de su interés. Narraba cómo conocía todas sus canciones y lo importantes que eran para ella.

Youseff, se mantuvo en movimiento durante toda la sesión y mostró interés por la dinámica inicial:

"Yo prefiero hacer actividades como la del principio, más de jugar" Parecía inquieto y cambiaba de lugar constantemente: "Es que me aburro. Tengo ganas de llegar a casa, ir a la mezquita y después jugar con el móvil hasta que me vaya a dormir”. [...] pregunté si se relacionaba con alguien para realizar estas actividades: "No, lo hago solo" Después de esto hizo un dibujo rápido en el que se representó a él mismo y lo mostró: "Este soy yo". Sin interpretaciones, resulta llamativo este acto en una actividad basada en "personas significativas para nosotras" (Alicia_NotasCampo_24.01)

En este evento se ha evidenciado la dificultad de los alumnos de explorar sus motivos personales dentro de la dinámica relacional de su contexto, a la vez que como algunos, como Abdu, construyen estrategias de resistencia (Ogbu, 1994). 


\section{EVENTO 4. IDENTIDADES EN DIÁLOGO}

En el taller de expresión teatral sobre fondos culturales hicimos una dinámica que nos llevó a conversar en torno a las identidades culturales y, específicamente, relativo a lo escolar-social y lo familiar. Durante esta sesión todas las personas que participamos éramos racializadas: tres estudiantes (Sara, Fatiha y Misbah) y dos investigadoras/educadoras (Yue y Ari). Empezamos la sesión con dinámicas teatrales a modo de calentamiento y seguidamente pasamos a realizar el "Eje de culturas". Esta actividad consistía en trazar una línea imaginaria en el suelo, situando en un extremo la cultura escolar/social y en el otro la cultura familiar. Empezando desde el punto medio, se trataba de moverse a lo largo de este eje en función de las siguientes frases que la dinamizadora va enunciando:

- Cómo te sientes cuando estás en la calle

- Cómo te sientes cuando estás en la escuela

- Cómo te sientes cuando hablas tu lengua materna en la calle

- Cómo te sientes cuando estás en la calle con tu familia

Después de cada enunciado, pasábamos a conversar y compartir reflexiones. Ari y Yue también participaban en la actividad, para tratar de enriquecer el debate. Durante la conversación participaron sobre todo Fatiha y Sara. Misbah, quien llegó hace un año a Barcelona, en todos los enunciados se situaba en la cultura familiar. Ella no mostraba tensiones o conflictos al respecto pero de sus intervenciones deducimos interés en participar, pues eran siempre para pedir más enunciados. En esta actividad la conversación se sucedió de forma fluida, y emergieron diferentes temas.

Fatiha y Sara hablan sobre lo cansado que es que la gente constantemente te diga que hables de una manera u otra y sobre el hecho de que la gente te defina "quién eres" cuando tu misma te sientes X o Y. (Yue_NotasCampo_31.01)

Esta actividad, consistente en promover que se identifiquen culturalmente, permite a Fatiha enunciar su malestar respecto al hecho de ser constantemente definida por los demás, y presuponer su cultura y lugar de procedencia basándose en la racialización y obviando su propio sentimiento. Es importante resaltar que esto no tiene que ver con un rechazo a la cultura con la que es identificada, pues ella llega a apropiársela:

\section{Antes me molestaba que me dijeran "mora” pero ahora ya no (Fatiha_NotasCampo_31.01)}

Se trata, en cambio, de una resistencia a ser etiquetada desde fuera, de un cansancio a que se la defina sin tener en cuenta cómo ella se siente. Fatiha muestra así rechazo a la diferenciación basada en la racialización (Buraschi y Aguilar-Idàñez, 2017) y reivindica el derecho a narrar su propia identidad (Taylor, 1996).

En la misma actividad, este derecho es reclamado por Sara de otra forma, en este caso, como resistencia a la imposición del modelo de asimilación cultural.

Ella expresa que no le gusta el catalán, no puede con él. Lo entiende pero no quiere hablarlo. Simplemente no puede. Y se agobia cuando las profesoras le empiezan a 
dar el discurso en catalán. Le digo que es normal y le pongo ejemplo de yo al no querer hablar en chino en espacio público porque se me asociaba a cosas negativas *lo que quería era ver si para ella hablar catalán era malo en tanto que traicionaba lo gitano*. (Yue_NotasCampo_31.01)

Yue ha identificado el rechazo de Sara al catalán y se aproxima a hablar sobre ello desde la propia experiencia para explorar si ella identifica hablar catalán como ceder ante la presión de la asimilación paya. Esta exploración de qué significaría traicionar a la propia cultura, desemboca en una conversación sobre afinidades y amistades, en la que Sara defiende su capacidad de decidir sus amistades fuera de intentos de control de su comunidad.

La gente me critica, por ejemplo, unas amigas gitanas que me dicen "pero por qué vas con marroquíes, etc.” y yo pues me da igual, yo voy con quien me siento bien y me da igual si eres marroquí, pakistaní o lo que sea. Me refiero que si me caes bien, pues ya está. (Sara_NotasCampo_31.01)

Hablamos sobre si hay solo una manera de ser gitana o ser marroquí, y cómo es o qué se presupone que es. Yue pone un ejemplo sobre lo que supone o se espera del ser china: la exigencia en hablar y escribir muy bien un idioma. Fatiha afirma y parece sentirse interpelada. Este tema ya emergió en una sesión anterior mientras realizaban el taller de expresión lingüística sobre fondos sociales.

Fatiha comenta que dentro de su familia el padre es la figura más cercana a ella, se considera bastante querida. Sin embargo, los domingos debe acudir por presión de este a clases de árabe escrito, situación que la agobia enormemente pues ya sabe hablarlo y no ve el sentido práctico de poder escribirlo. (Alicia_NotasCampo_17.01)

Volviendo a la sesión de los ejes culturales, Fatiha aportó una reflexión a la cuestión del ser de una cultura u otra que dio pie a la siguiente interacción:

Fatiha: También por presión de los padres, que te exigen eso.

Sara: Claro porque te quieren dar su cultura y quieren que seas igual que ellos.

Yue: Ay qué pesaos a veces, ¿que nos dejen un poco en paz no? Que cada una es diferente. (Fatiha y Sara se ríen) (Fatiha, Sara y Yue_NotasCampo_31.01)

Fatiha y Sara comparten experiencias personales en las cuales se sienten interpeladas entre ellas y se identifican, y mi intervención permite normalizar la situación, a la vez que baja el nivel de gravedad. El diálogo es entonces tanto relevante como con un matiz emocional positivo.

También hacemos énfasis en cómo en varias situaciones nos vamos ubicando en diferentes posiciones según cómo nos sentimos y recordamos la idea de que las personas directamente nos sitúan en el polo de "cultura familiar", que no se cuestionan que podamos estar también en el social-escolar. 


\section{CONCLUSIONES}

El análisis llevado a cabo sobre unos pocos eventos de una intervención todavía en curso nos muestra algunas potencialidades del uso de los FdI para la creación de un tercer espacio en el marco de la escuela intercultural. Este tercer espacio es un lugar donde el guion del profesorado y el del alumnado se cruzan, creando el potencial para que ocurra una interacción auténtica. El tercer espacio es una creación nueva, en la frontera entre el espacio escolar y el familiar/comunitario, a partir de la legitimación de los referentes culturales de los diversos participantes en la actividad (DiGiacomo y Gutiérrez, 2017).

Para ello ha resultado imprescindible el trabajo con grupos pequeños que permitan la confidencialidad, ya que un grupo grande no parece un espacio seguro que permita un clima de intimidad que permita hablar sobre aspectos personales. Es en grupos reducidos donde emerge un diálogo más fluido y se abordan temas más personales. En cambio, las dinámicas de este proyecto evidenciaron resultados favorables respecto a la comunicación en pequeños grupos, de los que han surgido espacios de confianza. Las narrativas identitarias apoyaban sus discursos en la producción artística y en el proceso fluían las interacciones y el diálogo sobre expectativas de futuro, ansiedades, vínculos sociales, aficiones.

Los eventos analizados muestran la posibilidad de descubrir y discutir la realidad cultural, así como la propia, a través del diálogo abierto por los FdI. Estos eventos se muestran relevantes para mostrar a los alumnos cosas sobre sí mismos que les resulta positivo descubrir, y contrastan con las expectativas iniciales respecto a no ser escuchados, lo que parece ser habitual en sus prácticas educativas cotidianas.

En el primer evento asistimos a un proceso de legitimación que abre la puerta a conectar el mundo personal y familiar con la práctica escolar. Frente a la resistencia a incorporar esta a la propia identidad, el diálogo generado por los fondos recogidos lleva a una elaboración identitaria del alumno, que reflexiona sobre su trayectoria personal. En el segundo evento se produce un diálogo auténtico sobre la noción de cultura a partir de las propias pertenencias y del contraste favorecido por un diálogo sin censuras. El tercer evento nos muestra la sutilidad de los procesos de asimilación cultural y de las actuaciones de resistencia a la misma. Nos muestra cómo las emociones juegan un papel central en los procesos de asimilación, y cómo las dinámicas de aceptación en las relaciones en la escuela son fundamentales en la generación de actuaciones de resistencia. Por último, en el cuarto evento se muestran tanto las potencialidades como las dificultades de un diálogo sobre las culturas que es en sí un diálogo entre culturas.

Adicionalmente, pero no menos importante, todos los eventos nos muestran lo inadecuado de las aproximaciones esencialistas a la diversidad cultural. Aunque en los diálogos de los alumnos hay constantes referencias a "payos", "moros"... haciéndose eco del lenguaje popular, cargado de connotaciones racistas, emergen como elementos clave de la identidad cultural aquellos que vinculan a los alumnos, a través de las emociones, a una red compleja formada por la familia, la comunidad, la religión, las amistades y la misma escuela.

\section{REFERENCIAS BIBLIOGRÁFICAS}

Bajtín, M.M. (2000). Yo también soy:(fragmentos sobre el otro). México: Taurus. Bruner, J. (1997). La educación, puerta de la cultura. Madrid: Visor. 
Estudios Pedagógicos XLV, $\mathrm{N}^{\circ}$ 1: 61-81, 2019

LOS FONDOS DE IDENTIDAD Y EL TERCER ESPACIO. UNA ESTRATEGIA DE LEGITIMACIÓN CULTURAL Y DIÁLOGO PARA LA ESCUELA INTERCULTURAL

Buraschi, D., y Aguilar-Idáñez, M.J. (2017). Herramientas conceptuales para un antirracismo críticotransformador. Tabula Rasa, (26), 171-191.

Cammarota, J. (2014). Misspoken in Arizona: Latina/o students document the articulations of racism. Equity \& Excellence in Education, 47(3), 321-333.

Cole, M. (2016). Designing for development: Across the scales of time. Developmental Psychology, 52(11), 1679-1689.

Connery, M.C., John-Steiner, V., \& Marjanovic-Shane, A. (Eds.). (2010). Vygotsky and creativity: A cultural-historical approach to play, meaning making, and the arts (Vol. 5). Peter Lang.

De Sousa Santos, B. (2003). Estado, democracia y globalización. La caída del Angelus Novus: Ensayos para una nueva teoría social, 125-273.

Díaz Barriga, F., López, E. y Vázquez, V. (2018). Exploración de los fondos de identidad en estudiantes de posgrado mediante una adaptación de la Multimetodología autobiográfica extendida (MAE). Papeles de Trabajo sobre Cultura, Educación y Desarrollo Humano, 14(3), 1-22.

DiGiacomo, D. \& Gutiérrez, K. (2017). Seven chilis: making visible the complexities in leveraging cultural repertories of practice in a designed teaching and learning environment. Pedagogies: An International Journal, 12(1), 41-57.

Engeström, Y. (2011). From design experiments to formative interventions. Theory \& Psychology. 21(5), 598-628

Esteban-Guitart, M. (2012). La Multi-metodología Autobiográfica Extendida (MAE). Una estrategia cualitativa para estudiar la identidad, los fondos de conocimiento y las formas de vida. Revista Electrónica de Metodología Aplicada, 17(2), 51-64.

Esteban-Guitart, M. \& Moll, L.C. (2014). Funds of Identity: a new concept based on the Funds of Knowledge approach. Culture \& Psychology, 20(1), 31-48.

González, N., Moll, L. \& Amanti, C. (Eds.). (2005). Funds of knowledge: Theorizing practices in households, communities, and classrooms. New York, NY: Routledge.

Gutiérrez, K. \& Vossoughi, S. (2010). Lifting off the ground to return anew: Mediated praxis, transformative learning, and social design experiments. Journal of Teacher Education, 61(1-2), $100-117$.

Lalueza, J., Sánchez-Busqués, S. \& García-Romero, D. (2019). Following the trail of the 5th Dimension: University-community partnership to design educational environments. Mind, Culture \& Activity.

Llopart, M. \& Esteban-Guitart, M. (2016). Funds of knowledge in 21st century societies: inclusive educational practices for under-represented students. A literature review. Journal of Curriculum Studies, 50(2), 145-161.

Matusov, E., Smith, M., Soslau, E., Marjanovic-Shane, A., \& Von Duyke, K. (2016). Dialogic education for and from authorial agency. Dialogic Pedagogy: An International Online Journal, 4, 162-197.

Matusov, E., Marjanovic-Shane, A. \& Gradovski, M. (2019). Dialogic pedagogy and polyphonic research: Bakhtin by and for educators: Palgrave Macmillan.

Moreno, A. (2016). La mediación artística : Arte para la transformación social, la inclusión social y el trabajo comunitario. Barcelona: Ediciones Octaedro.

Nilson, M. \& Nocon, H. (Eds.) (2005). School of Tomorrow.Developing Expansive Learning Environments. London: Peter Lang.

Poveda, D. (2001). La educación de las minorías étnicas desde el marco de las continuidadesdiscontinuidades familia-escuela. Gazeta de Antropología, 17, 17-31.

Sebastián, C., Gallardo, G. y Calderón, M. (2016). Sentido identitario de la formación. Una propuesta para articular el desarrollo de la identidad y el aprendizaje en contextos educativos. Papeles de Trabajo sobre Cultura, Educación y Desarrollo Humano, 12(3), 2-9.

Subero, D., Brito, L. y Esteban-Guitart, M. (2017). La incorporación de los fondos de conocimiento e identidad en la escuela normal de Texcoco, México. Las creencias de los docentes en formación, 


\section{$2,9-25$.}

Subero, D., Vila, I. \& Esteban-Guitart, M. (2015). Some contemporary forms of the funds of knowledge approach. Developing culturally responsive pedagogy for social justice. International Journal of Educational Psychology, 4(1), 33-53.

Taylor, C. (1996). Identidad y reconocimiento. Revista Internacional de Filosofía Política, 7, 10-19. Vygotski, L.S. (2007). La imaginación y la creatividad en la infancia. La Educación Artística y Creativa. Escritos sobre arte y educación creativa de Lev S. Vygotski. Madrid: Fundación Infancia y Aprendizaje.

Willig, C. (2013). Introducing Qualitative Research in Psychology. New York: Open University Press. Zipin, L. (2009). Dark funds of knowledge, deep funds of pedagogy: Exploring boundaries between lifeworlds and schools. Discourse: Studies in the Cultural Politics of Education, 30, 317-331. 
